\title{
Production of soluble and functional engineered antibodies in Escherichia coli improved by FkpA
}

\author{
Zhong Zhang, Li-ping Song, Min Fang, Fei Wang, Dan He, Rui Zhao, Jing Liu, \\ Zhi-yong Zhou, Chang-cheng Yin, Qing Lin, and Hua-liang Huang
}

BioTechniques 35:1032-1042 (November 2003)

\begin{abstract}
Overproduction of genetically engineered antibodies, such as single-chain antibodies (scAbs) in Escherichia coli often results in insoluble and inactive products known as inclusion bodies. We now report that fusion or co-expression of FkpA, the E. coli periplasmic peptidyl-prolyl-isomerase with chaperone activity, substantially improves soluble and functional expression of scAbs. Anti-human bladder carcinoma scAb $(P G)$ and anti-human CD3×anti-human ovarian carcinoma-bispecific scAb (BH1) were fused with FkpA on the pTMF-based plasmid and expressed in E. coli. More than half of the amount of each expressed fusion protein FkpA-PG or FkpA-BH1 was soluble. In addition, the fusion protein cellulose-binding domain from Cellulomonas fimi (CBD)-PG and anti-human CD3×antihuman CD28Xanti-human ovarian carcinoma-trispecific scAb (TRI) fused to the pelB (a signal peptide from pectate lysase B of a Bacillus sp.) signal sequence were co-expressed with FkpA under the control of the T7 promoter. A substantial portion of the co-expressed CBD-PG or TRI was soluble. Furthermore, PG, BHI, and TRI were biologically active as judged by ELISA and in vitro cytotoxicity assay. These results suggest that overexpression of FkpA should be useful in expressing heterologous proteins in E. coli.
\end{abstract}

\section{INTRODUCTION}

Genetically engineered antibodies and their fragments produced in Escherichia coli have a great potential as specific targeting reagents, both in scientific research and clinical trials $(1,2)$. There has been growing interest especially in the single-chain antibody $(\mathrm{scAb})$ format of engineered antibodies, particularly for in vivo diagnostic and therapeutic applications because of its small size. However, one major factor that can limit the applicability of scAbs is the ability to produce them in large amounts as soluble and functional proteins. Actually, scAbs are often prone to form insoluble aggregates known as inclusion bodies when expressed in E. coli, especially at high level, resulting in low active yields. Although the expression of proteins as inclusion bodies leads to larger amounts of target proteins, the production of biologically functional products is stymied by the laborious procedures $(3,4)$. Many strategies have been proposed to obtain improved solubility in the past years (5-14). However, the application of signal sequences or different culture conditions to a variety of engineered antibodies within our laboratory has met with little success (15). Therefore, as the optimization of engineered antibody expression still tends to proceed on a case-by-case basis, a generic system is desirable to improve engineered antibody expression as soluble and active products in E. coli.

FkpA, a periplasmic peptidyl-prolyl cis, trans-isomerase (16), is one of the heat shock proteins that are inducible in response to accumulation of misfolded proteins in E. coli (16-18). Missiakas et al. found that FkpA decreased the sigma E-dependent response constitutively induced by misfolded proteins and proposed that FkpA plays an active role either as a folding catalyst or as a chaperone in E. coli (18). Further investigation showed that FkpA has two activities, the cis, trans-isomerase activity and the chaperone-like activity (19-21). A poorly folded single-chain variable fragment $(\mathrm{scFv})$ displayed on filamentous phage and a defective- folding variant of the maltose-binding protein, MalE31, were produced in biologically active form in E. coli functionally assisted by co-expression of $\operatorname{FkpA}(21,22)$.

Because all the engineered antibodies constructed in our laboratory were insoluble when expressed in E. coli, we sought to identify whether overexpression of FkpA would be of benefit to the expression of engineered antibodies of interest as soluble and functional forms. We have constructed various vectors for the fusion expression or co-expression of FkpA with engineered antibodies anti-human bladder carcinoma scAb (PG) and its fusion protein cellulose-binding domain from Cellulomonas fimi (CBD)-PG, anti-human CD3×anti-human ovarian carcinomabispecific $\mathrm{scAb}$ (BH1), and anti-human CD3×anti-human CD28×anti-human ovarian carcinoma-trispecific scAb (TRI). Our results demonstrate conclusively that fusion expression or co-expression of FkpA is sufficient for soluble and functional expression of engineered antibodies in E. coli. 
Table 1. Plasmids Used in this Study

\begin{tabular}{|c|c|c|}
\hline Plasmid & Relevant Characteristics & $\begin{array}{l}\text { Reference or } \\
\text { Source }\end{array}$ \\
\hline pTMF & $5.3 \mathrm{~kb}, \mathrm{Km}^{\mathrm{r}}$, T7 promoter & Our laboratory \\
\hline pTCM & $5.3 \mathrm{~kb}, \mathrm{Km}^{\mathrm{r}}$, c-myc tag, T7 promoter & Our laboratory \\
\hline p TP1 & $6.0 \mathrm{~kb}, p g-c-m y c$ & Our laboratory \\
\hline pTFP1 & $6.8 \mathrm{~kb}, p g-c-m y c$ fused to $f k p A$ & This study \\
\hline pTMFBH1 & $6.9 \mathrm{~kb}$, bh1-c-myc & Our laboratory \\
\hline pTFBH1 & $7.7 \mathrm{~kb}, b h 1-c-m y c$ fused to $f k p A$ & This study \\
\hline pTBNP1 & $6.5 \mathrm{~kb}, c b d$ fused to $p g-c-m y c$ & Our laboratory \\
\hline pTBNP1F & $\begin{array}{l}7.3 \mathrm{~kb}, c b d \text { fused to } p g-c-m y c \text { and } \\
\text { co-expressed with } f k p A\end{array}$ & This study \\
\hline pTTRI & $7.4 \mathrm{~kb}$, tri & Our laboratory \\
\hline pTTRIF & $8.2 \mathrm{~kb}$, tri, co-expressed with $f k p A$ & This study \\
\hline
\end{tabular}

\section{MATERIALS AND METHODS}

\section{Reagents}

Mouse anti-c-myc monoclonal antibody (cell line 9E10) was purchased from Santa Cruz Biotechnology (Santa Cruz, CA, USA). Goat anti-mouse immunoglobulin $G$ horseradish peroxi- dase (IgG-HRP) and protein markers were purchased from Sino-American Biotechnology Company (Beijing, China). Anti-CD3 monoclonal antibody was purchased from Wuhan Institute of Biological Products (Wuhan, China). Anti-CD28 monoclonal antibody and rhCD28/FcChimera, recombinant human CD28 extracellular domain fused
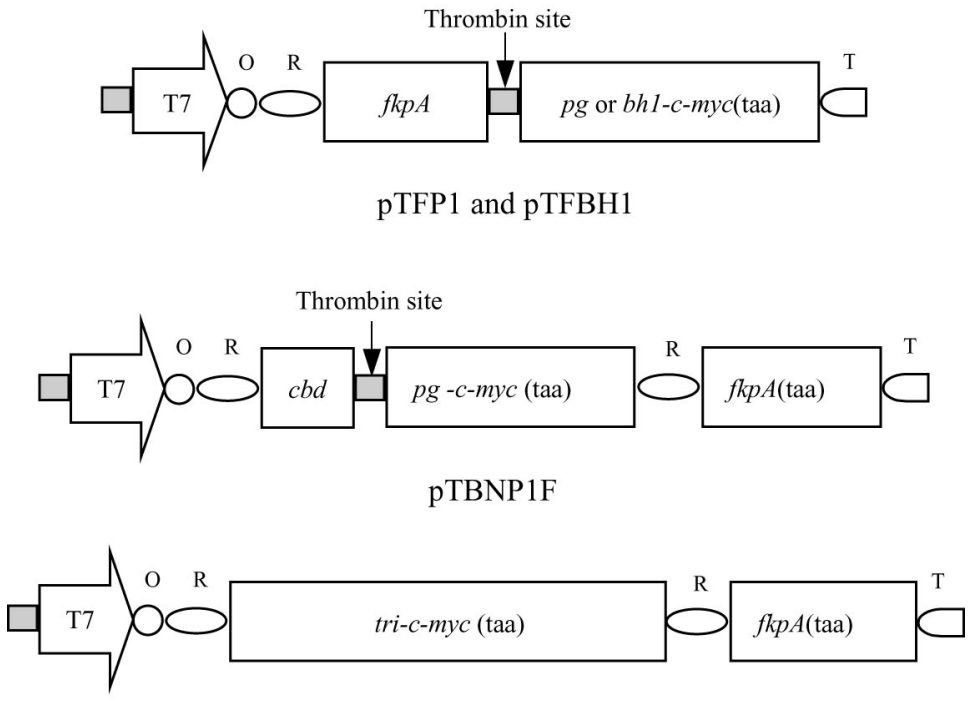

pTTRIF

Figure 1. pTMF-based expression plasmids. Organization of $f k p A$ and $p g, b h l, c b d-p g$, and tri is schematically shown here. $p g, b h l, c b d-p g$, and tri genes were joined with $f k p A$, respectively, on the pTMF under the control of the T7 promoter and the lac operator. T7, the T7 promoter; $\mathrm{O}$, the lac operator; R, ribosome binding site (RBS); T, the T7 transcription terminator; taa, stop codon; c-myc, a short 11-amino acid tag.

to human $\mathrm{IgG}_{1} \mathrm{Fc}$, were products of R\&D System (Minneapolis, MN, USA). Anti-ovarian carcinoma $\mathrm{scFv}$ was prepared by renaturing and purifying the inclusion body expressed in E. coli. Restriction endonucleases, $\mathrm{T}_{4}$ DNA ligase, LA Taq DNA polymerase, and other modification enzymes were purchased from TaKaRa Biotechnology (Dalian), Ltd. (Dalian, China). $p f u$ DNA polymerase was a product of Bioasia Company (Shanghai, China). Prestained protein markers were purchased from New England Biolabs (Beverly, MA, USA).

Human bladder carcinoma cell line BIU87, human ovarian carcinoma cell line SKOV3, and human T lymphoma cell line Jurkat, on the surface of which CD3 was overexpressed, were from our laboratory.

MTT was purchased from Sigma (St. Louis, MO, USA). All other chemicals were of analytical grade.

\section{Bacterial Strains and Plasmids}

E. coli $\mathrm{DH} 5 \alpha$ and Top 10 were used as the host strains for cloning and maintenance of plasmids throughout the experiments. E. coli BL21 Star (DE3) was purchased from Invitrogen (Carlsbad, CA, USA) and used as the host for expression of all the $\mathrm{T} 7$ promoter-based vectors constructed in this study.

The plasmid pTMF was constructed in our laboratory (15) and used as the basic vector for construction of all the expression plasmids in this study. The plasmid pTCM, constructed previously by inserting the complete coding sequence of c-myc (33 bp) into the multicloning site of pTMF (15), was used as a control in ELISA of expressed target proteins against specific antigens. Plasmids pTP1 (15) and pTBNP1 (unpublished) were constructed previously and used for expression of $\mathrm{PG}$ and its fusion protein CBD-PG. Plasmids pALMBH1 and pTTRI were constructed in our laboratory (unpublished data) and used for expression of BH1 and TRI, respectively. All plasmids constructed and used in this study were diagrammatically shown in Figure 1 and summarized in Table 1.

For the facile detection of the expressed scAbs against specific antigens, the $\mathrm{C}$ terminus of each of the scAbs mentioned in this study contains a c-myc 
tag, which can be detected by mouse anti-c-myc monoclonal antibody.

\section{Isolation of the $f k p A$ Open Reading Frame}

fkpA genes [complete coding sequence with or without a ribosome binding site (RBS)] were isolated by PCR with $p f u$ polymerase, using $E$. coli strain K12 chromosomal DNA as templates. The complete $f k p A$ was amplified as follows: the forward primer was 5'-AAATCACTGTTTAAAGTAACG-3'. The reverse primer, 5'-GCGAGCTCTTTTTTAGCAGAATCTGCGG-3', was designed to contain a SacI site (underlined). The PCR product was digested with SacI, purified, and cloned into the NcoI (blunted by the Klenow fragment of $E$. coli DNA polymerase I)-SacI site of pTMF, yielding plasmid pTFA (Figure 1). Similarly, $r b s-f k p A$ was amplified using the following primers. The forward primer was 5'-GAATGGCATATGAATAATTTTGTTTAACTTTAAGAAGGAGATATATCCATGAAATCACTGTTTAAAGTAAC-3' and was designed to contain a $\mathrm{Nde}$ I site (underlined) and an RBS site (bolded). The reverse primer was 5'-GCGGATCCTTATTTTTTAGCAGAATCTGC-3' containing a BamHI site (underlined). The PCR product was digested with NdeI and BamHI, purified, and cloned into the same sites of pTMF, yielding pRFA (Figure 1).

The sequences of all the amplified genes were verified by DNA sequencing.

\section{Construction of the FkpA Fusion and Co-Expression Vectors}

For construction of the FkpA-PG fusion vector, the $\mathrm{BglII}-\mathrm{SacI}$ fragment of pTFA was inserted into the BglII-SacI site of pTP1 to yield pTFP1. For construction of FkpA-BH1 fusion vector, the bispecific scAb BH1 was cloned from plasmid pALMBH1 by PCR using specific primers. The forward primer was 5'-CCGCTCGAGGTGCAGCTGCAGGAGTCTGG-3' designed to contain an $X h$ oI site (underlined), and the reverse primer was 5'-TTACTGCAGTTATCAGTTCAGGTCTTCTTCAG-3'. $p f u$ polymerase was used in the PCR, and the products were recovered, digested with XhoI, and ligated into the XhoI-NdeI (blunted by the Klenow fragment of $E$. coli DNA polymerase I) site of pTFA to yield pTFBH1.

For construction of FkpA co-expression vector to co-express CBD-PG, the $B g l$ III-XhoI fragment of pTBNP1 was inserted into the $B g l \mathrm{II}-\mathrm{X} h o \mathrm{I}$ site of pTRFA, and the resulting plasmid was designated pTBNP1F and used to co-express FkpA with fusion protein CBD-PG. For construction of FkpA co-expression vector to co-express trispecific scAb TRI, two specific primers were used to isolate the trispecific $\mathrm{scAb}$ gene by PCR from plasmid pTRI, which was constructed previously (Song et al., 
unpublished). The forward primer was 5'-CATCACCATCACCATCACCCGTGCCGTCCATGTACTCAC-3', and the reverse primer was $5^{\prime}$-TTACGGGCAAGGTGGACAAGT-3’. To ensure the efficiency of PCR in cloning the long fragment TRI, LA Tag DNA polymerase was used. The PCR product was recovered and ligated into the $\mathrm{NcoI}$ site (blunted by the Klenow fragment of $E$. coli DNA polymerase I) of pTRFA. The resulting plasmid was designated pTTRIF. Each subset of the genes obtained above was placed under the control of the $\mathrm{T} 7$ promoter and the lac operator on pTMF-based vectors. The sequences of all the amplified genes were verified by DNA sequencing. All the pTMF-based vectors constructed in this study are diagramed in Figure 1.

\section{Culture Conditions, Protein Expression, and Analysis}

All the expression plasmids were used to transform E. coli BL21 Star

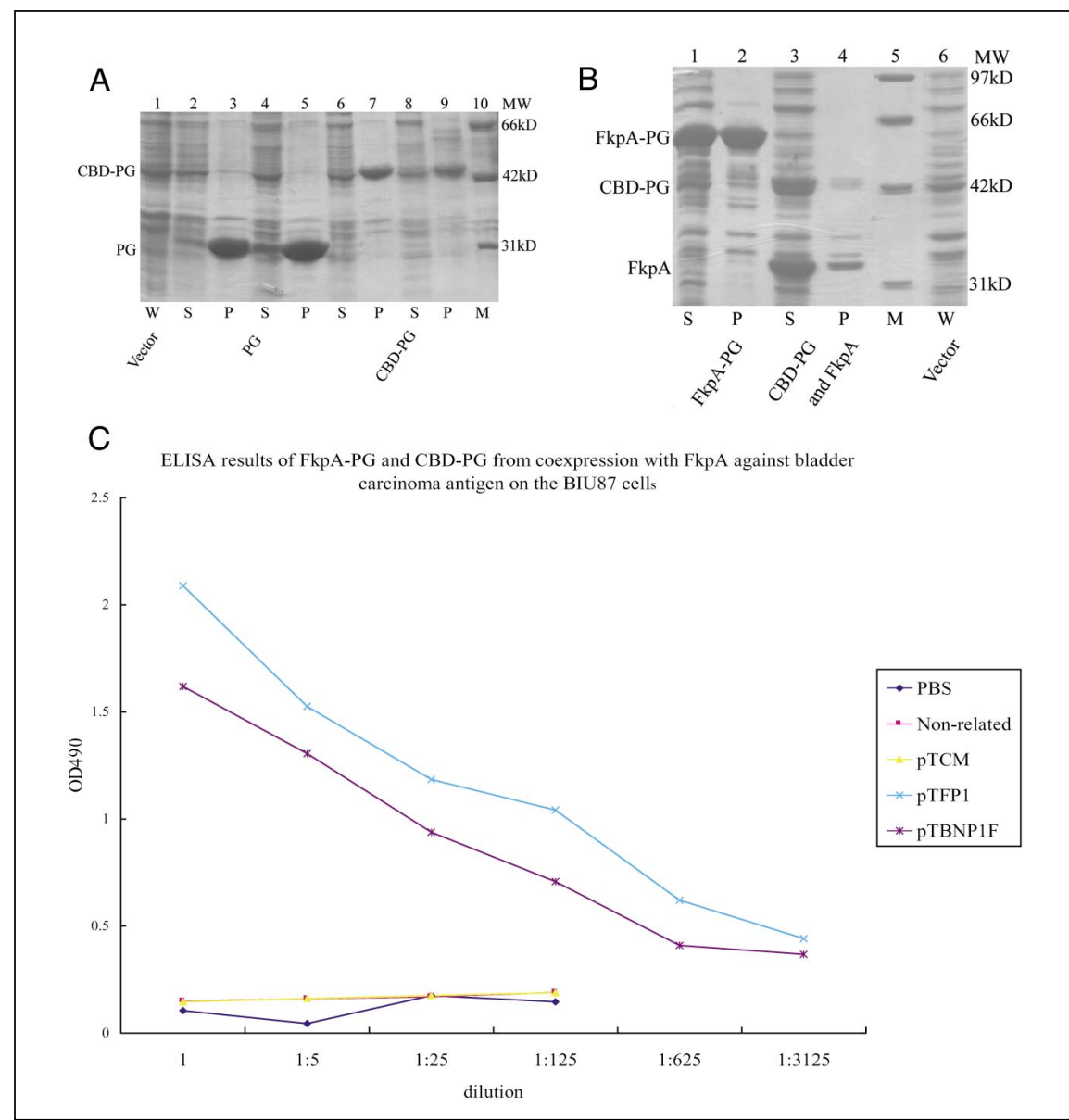

Figure 2. FkpA markedly improves the expression of scAb PG and its fusion protein CBD-PG as the soluble and biologically active forms in Escherichia coli. (A and B) Sodium dodecyl sulfate polyacrylamide gel electrophoresis (SDS-PAGE) analysis of PG and CBD-PG (A) without or (B) with fusion or co-expression of FkpA in E. coli. (A) Lane 1, the whole-cell proteins (W) of E. coli cells harboring pTMF (empty vector); lanes 2 and 3, supernatant (S) and pellet (P), respectively, of $E$. coli cells harboring pTP1 induced at $37^{\circ} \mathrm{C}$; lanes 4 and 5 , supernatant and pellet, respectively, of $E$. coli cells harboring pTP1 induced at $30^{\circ} \mathrm{C}$; lanes 6 and 7, supernatant and pellet, respectively, of E. coli cells harboring pTBNP1 induced at $37^{\circ} \mathrm{C}$; lanes 8 and 9 , supernatant and pellet, respectively, of $E$. coli cells harboring pTBNP1 induced at $30^{\circ} \mathrm{C}$; lane 10 , molecular mass markers $(\mathrm{M} ; \mathrm{kDa})$. (B) Lanes 1 and 2 , supernatant and pellet, respectively, of $E$. coli cells harboring pTFP1; lanes 3 and 4, supernatant and pellet, respectively, of E. coli cells harboring pTBNP1F; lane 5, molecular mass markers; lane 6, the whole-cell proteins of E. coli cells harboring pTMF. (C) ELISA results of FkpA-PG and CBD-PG coexpressed with FkpA against the membrane antigens of BIU87. Phosphate-buffered saline (PBS) and nonrelated scAb-c-myc were used as controls. The sonication supernatants of $E$. coli cells carrying respective plasmid pTCM, pTFP1, and pTBNP1F were tested. CBD, cellulose-binding domain from Cellulomonas fimi; PG, anti-human bladder carcinoma scAb; scAb, single-chain antibody.

(DE3). BL21 Star (DE3) transformed cells were grown in LB medium (23) supplemented with antibiotics. Cultures were induced at an $\mathrm{A}_{600}=0.8$ using $0.4 \mathrm{mM}$ isopropyl- $\beta$-D-thiogalactopyranoside (IPTG). Typically, after several hours of induction, the induced cells from $1 \mathrm{~mL}$ of culture broth were harvested by centrifugation, resuspended in phosphate-buffered saline (PBS), and then treated by sonication. After centrifugation at $8000 \times g$ for $10 \mathrm{~min}$ at $4^{\circ} \mathrm{C}$, the supernatant was taken as soluble fraction. The pellet was taken as insoluble fraction and resuspended in PBS. If needed, whole-cell proteins were prepared separately by harvesting $1 \mathrm{~mL}$ of culture broth directly with centrifugation. Thirty microliters of each of the above fractions and the wholecell proteins were analyzed by sodium dodecyl sulfate polyacrylamide gel electrophoresis (SDS-PAGE), followed by visualization with Coomassie ${ }^{\circledR}$ Brilliant Blue staining, and scanning quantitative analysis by spot density using the marker proteins as control.

\section{ELISA for scFvs Activity and Western Blot Analysis}

ELISAs were carried out in order to evaluate the antigen-binding activities of the soluble engineered antibodies PG, BH1, and TRI expressed in E. coli under the assistance of FkpA. Membrane antigens of BIU87 cells, Jurkat cells and SKOV3 cells, and CD28 pure antigen (rhCD28/FcChimera) were diluted in $0.1 \mathrm{M} \mathrm{NaCO}_{3}-\mathrm{NaHCO}_{3}$ buffer, $\mathrm{pH}$ 9.6, and the final concentration of each antigen was adjusted to $10 \mu \mathrm{g} /$ mL. ELISA plates (NUNC, Roskilde, Denmark) were coated overnight at $4^{\circ} \mathrm{C}$ with each of the above membrane antigens, respectively. After washing three times with PBS with Tween ${ }^{\circledR} 20$ (PBST) and three times with PBS, the plates were blocked for $2 \mathrm{~h}$ at $37^{\circ} \mathrm{C}$ with $200 \mu \mathrm{L}$ of $1 \%$ bovine serum albumin (BSA)-PBS and then washed with PBST and PBS, respectively. A total of $100 \mu \mathrm{L}$ supernatant containing expressed target proteins (PG, BH1, or TRI) was applied to the blocked ELISA wells and incubated for $2 \mathrm{~h}$ at $37^{\circ} \mathrm{C}$. PBS or supernatants from $E$. coli cells harboring empty vector pTMF or pTCM as negative controls, were also 
added and incubated for $2 \mathrm{~h}$ at $37^{\circ} \mathrm{C}$. After the plates were washed, $100 \mu \mathrm{L}$ mouse anti-c-myc monoclonal antibody were added and incubated for $1 \mathrm{~h}$ at $37^{\circ} \mathrm{C}$. For detection, $100 \mu \mathrm{L}$ of goat anti-mouse IgG-HRP antibody were used and incubated for another $1 \mathrm{~h}$ at $37^{\circ} \mathrm{C}$. The development was carried out with a total of $100 \mu \mathrm{L}$ o-phenylenediamine (OPD) solution $(6 \mathrm{mg} / \mathrm{mL}$ OPD, $1 \% \mathrm{H}_{2} \mathrm{O}_{2}$ ) for $15-20 \mathrm{~min}$ at room temperature, and signals were read at $490 \mathrm{~nm}$ with Universal Microplate Reader Elx800 (Bio-Tek Instruments, Winooski, VT, USA) after stopping the reaction with $50 \mu \mathrm{L} 2 \mathrm{M} \mathrm{H}_{2} \mathrm{SO}_{4}$.

Immunodetection by Western blot analysis was performed as described (23). Briefly, proteins from sonication supernatants were subjected to SDS-PAGE (12\% polyacrylamide gel). Prestained protein markers were used as the standard. Electrotransfer of proteins to nitrocellulose filters $(0.45 \mu \mathrm{m}$; Pall-Gelman Sciences, Ann Arbor, MI, USA) was performed in the transfer buffer $(39 \mathrm{mM}$ glycine, $40 \mathrm{mM}$ Tris-base, $0.03 \%$ SDS, $20 \%$ methanol) by using Hoefer ${ }^{\mathrm{TM}}$ TE 22 Mighty Small Transphor Tank Transfer Unit (Amersham Biosciences, Piscataway, NJ, USA) at constant current $400 \mathrm{~mA}$ for 1 $\mathrm{h}$, and then the filter was blocked with 2\% low-fat dry milk in TBS (TBS: 100 $\mathrm{mol} / \mathrm{L}$ Tris-HCL, $\mathrm{pH} 7.5,0.9 \% \mathrm{NaCl}$ ) at room temperature for about $1 \mathrm{~h}$. Then the filter was washed with TBST (TBS containing $0.1 \%$ Tween 20). Mouse anti-c-myc monoclonal antibody was added, and the filter was incubated for $1 \mathrm{~h}$ at room temperature. Next, goat anti-mouse IgG-HRP antibody was added, and the filter was incubated for another hour at room temperature. The peroxidase activity was developed with a solution of diaminobenzidine (DAB; $0.6 \mathrm{mg} / \mathrm{mL}$ ) in $20 \mathrm{mM}$ Tris-base, $0.5 \mathrm{M}$ $\mathrm{NaCl}$-buffered saline, and $0.1 \% \mathrm{H}_{2} \mathrm{O}_{2}$.

\section{In Vitro Cytotoxicity Assay for Trispecific scAb Co-Expressed with FkpA}

Human peripheral blood mononuclear cells (PBMCs) from healthy donors were isolated using Ficoll ${ }^{\circledR}$ density gradient centrifugation, and monocytes/macrophages were depleted using glass adherence incubated at $37^{\circ} \mathrm{C}, 5 \% \mathrm{CO}_{2}$ for $2 \mathrm{~h}$. A 96-well flatbottom plate was coated with target tumor cells SKOV3 and incubated overnight to prepare cell monolayers. The effector cells (PMBCs) were added to the tumor cell monolayers at the appropriate effector cells/tumor cells $(\mathrm{E} / \mathrm{T})$ ratios. Different dilutions of supernatants containing TRI were added to the plate, and then the plate was incubated at $37^{\circ} \mathrm{C}, 5 \% \mathrm{CO}_{2}$ for $72 \mathrm{~h}$. After PBMCs were removed by washing, MTT solution $(0.5 \mathrm{mg} / \mathrm{mL})$ was added, and the plate was incubated for another $4 \mathrm{~h}$. The MTT solution was then removed, and the crystals of formazan were dissolved in dimethyl sulfoxide (DMSO) at room temperature. The results were calculated based on the means of absorbance obtained from three wells according to the following formula:

$$
\% \text { lysis }=100 \times(\mathrm{C}-\mathrm{E}) /(\mathrm{C}-\mathrm{B})
$$

where $\mathrm{C}$ is the absorbance reading of the cells with target cells (control), B is the background, and $\mathrm{E}$ is the absorbance reading of adherence tumor cells remaining in the wells after co-incubation with PBMCs (24).

\section{RESULTS}

\section{PG, CBD-PG, BH1, and TRI Always Aggregate as Inclusion Bodies When Expressed in E. coli}

Although the induction conditions were optimized for efficient expression of the scAbs constructed in our laboratory, scAb PG and its fusion protein CBD-PG were totally insoluble in $E$. coli BL21 Star (DE3) (Figure 2A). For facilitating the secretion of expressed proteins, the mature $p g$ gene was fused to a signal peptide stII (a signal peptide from E. coli thermostable enterotoxinII) (25) or CBD (26), which contains a pow-
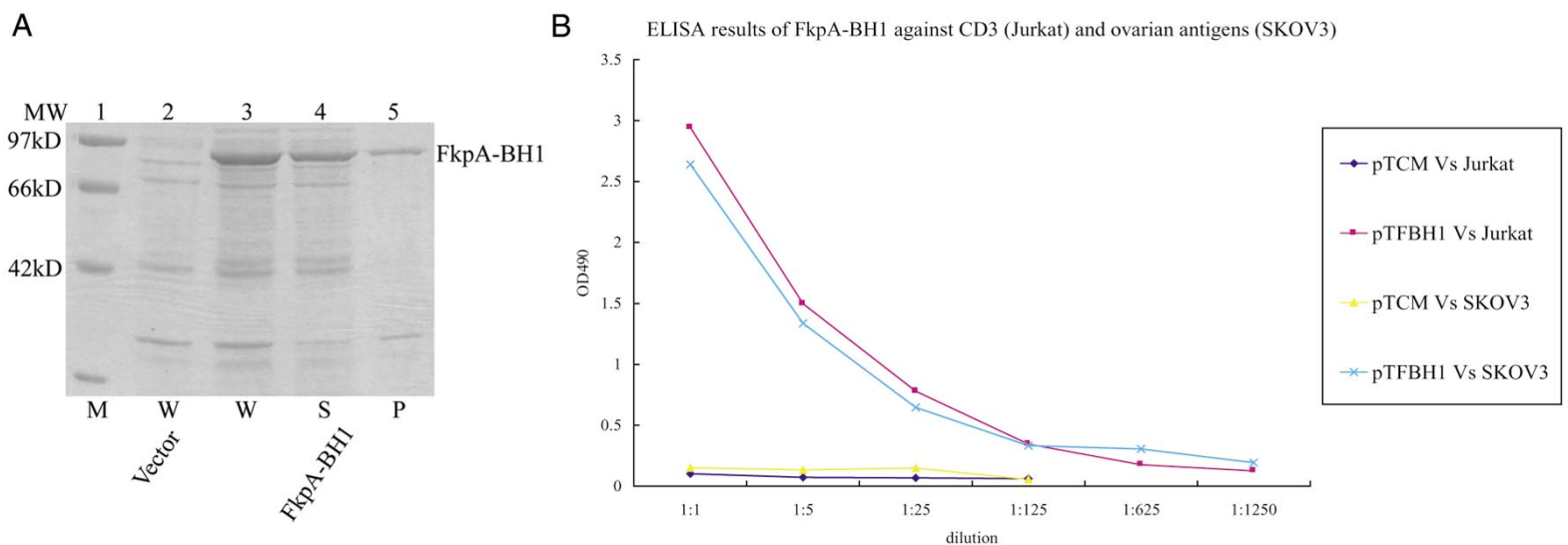

Figure 3. The fusion protein FkpA-BH1 was expressed as the soluble and biologically active form in Escherichia coli. (A) Sodium dodecyl sulfate polyacrylamide gel electrophoresis (SDS-PAGE) analysis of FkpA-BH1 expressed in E. coli. Lane 1, molecular mass markers (M); lane 2, the whole-cell proteins (W) of E. coli cells harboring pTMF; lanes 3, 4, and 5, the whole-cell proteins, supernatant (S) and pellet (P), respectively, of E. coli cells harboring pTFBH1. (B) ELISA results of FkpA-BH1 against both CD3 antigen from Jurkat and tumor cell antigens from SKOV3. pTCM vs. Jurkat and pTCM vs. SKOV3: the sonication supernatant from E. coli cells carrying empty vector pTCM was used against both the antigen from Jurkat (CD3) and SKOV3. pTFBH1 vs. Jurkat and pTFBH1 vs. SKOV3: the sonication supernatant from E. coli cells carrying pTFBH1 was used against both the antigen from Jurkat (CD3) and SKOV3. BH1, anti-human CD3×anti-human ovarian carcinoma-bispecific scAb; scAb, single-chain antibody. 
erful signal peptide, to yield expression vector pTP1 (15) and pTBNP1 (unpublished). However, no soluble products were observed as shown in Figure 2. Under the same conditions as mentioned above, the expressed BH1 existed as inclusion bodies (data not shown), and most of the expressed TRI existed as insoluble form (see Figure 4A).

\section{Effects of FkpA Fusion or Co-Expression on the Soluble Production of Engineered Antibodies in E. coli}

To achieve large amounts of soluble and biologically active scAbs, the FkpA-based fusion and co-expression vectors pTFP1, pTFBH1, pTBNP1F, and pTTRIF were constructed. In contrast to separate expression, most part of each $\mathrm{scAb}$ or its fusion protein FkpA-PG，FkpA-BH1，CBD-PG， or TRI was soluble after sonication of the whole cells analyzed by SDS-PAGE (Figures 2B, 3A, and 4A), and the soluble portion of FkpA-PG, FkpA-BH1, or CBD-PG accounts for $50 \%, 70 \%$,
A
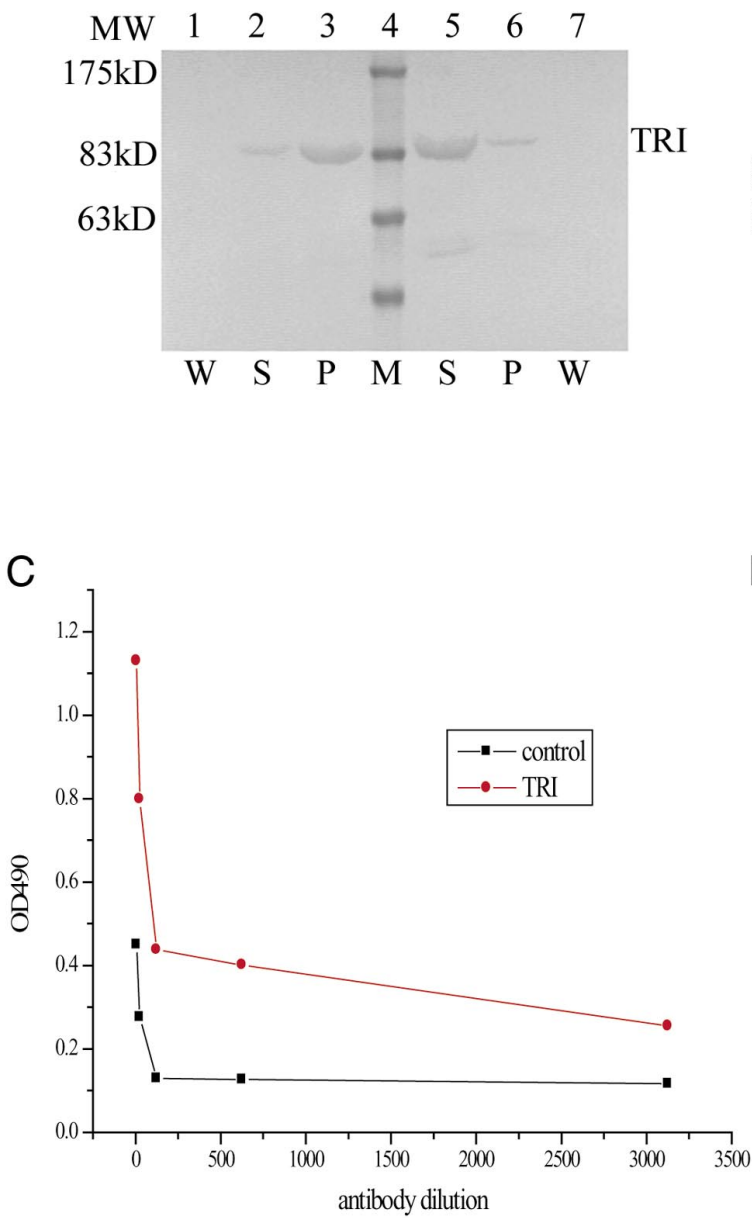
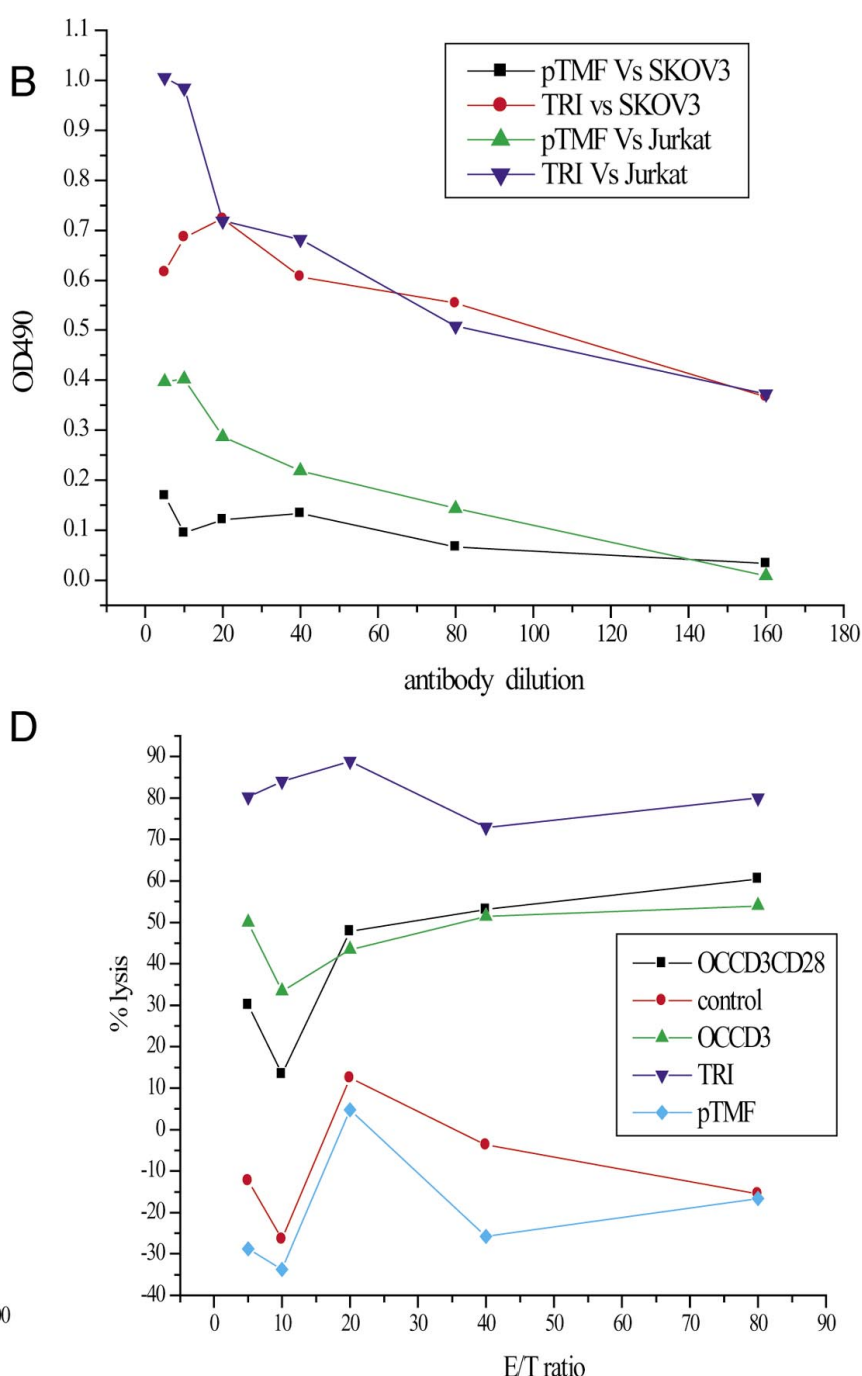

Figure 4. FkpA markedly improves the expression of trispecific scAb TRI as the soluble and biologically active form in Escherichia coli. (A) Results of Western blot analysis of TRI. Lanes 1 and 7, the whole-cell proteins (W) of E. coli cells harboring pTMF, and the uninduced $E$. coli cells harboring pTTRIF, respectively; lanes 2 and 3, supernatant (S) and pellet (P), respectively, of E. coli cells harboring pTTRI; lane 4, molecular mass markers (M); lanes 5 and 6, supernatant and pellet, respectively, of $E$. coli cells harboring pTTRIF. (B) ELISA results of TRI co-expressed with FkpA against CD3 molecules on Jurkat cells and tumor antigens on SKOV3, respectively. pTMF vs. SKOV3 and pTMF vs. Jurkat: supernatant of $E$. coli cells harboring pTMF against tumor antigens from SKOV3 and CD3 molecules from Jurkat, respectively; TRI vs. SKOV3 and TRI vs. Jurkat: supernatant of $E$. coli cells harboring pTTRIF against tumor antigens from SKOV3 and CD3 molecules from Jurkat, respectively. (C) ELISA results of TRI co-expressed with FkpA against CD28 antigen. TRI and control stand for supernatants of induced E. coli cells harboring pTTRIF and induced $E$. coli cells harboring pTMF, respectively. (D) TRI-mediated in vitro cytotoxicity against tumor cell SKOV3. OCCD3CD28: anti-ovarian scAb, anti-CD3 monoclonal antibody, and anti-CD28 monoclonal antibody were used simultaneously as positive control; OCCD3: anti-ovarian scAb and anti-CD3 monoclonal antibody were used simultaneously; control: RPM1640 culture medium was used as negative control; TRI: the sonication supernatant of $E$. coli cells carrying pTTRIF was used to evaluate the in vitro cytotoxicity of TRI co-expressed with FkpA; pTMF: the sonication supernatant of E. coli cells harboring empty vector pTMF was used as a control. scAb, single-chain antibody; TRI, anti-human CD3×antihuman CD28×anti-human ovarian carcinoma-trispecific scAb. 
Table 2. Comparison of Induced Soluble Protein Contents in E. coli BL21 Star (DE3) Harboring Various Constructs Used in this Study ${ }^{\mathrm{a}}$

\begin{tabular}{|c|c|c|c|}
\hline Plasmid & Target Protein & $\begin{array}{c}\text { Induction } \\
\text { Condition } \\
\left({ }^{\circ} \mathrm{C}\right)\end{array}$ & $\begin{array}{c}\text { Soluble Portion } \\
\text { in Total Target } \\
\text { Proteins } \\
\text { (\%) }\end{array}$ \\
\hline pTP1 & $P G$ & 37 & 14 \\
\hline pTFP1 & FkpA-PG & 37 & 50 \\
\hline pTBNP1 & CBD-PG & 37 & 9 \\
\hline pTBNP1F & $\begin{array}{l}\text { CBD-PG, co-expressed } \\
\text { with FkpA }\end{array}$ & 37 & 81 \\
\hline pTMFBH1 & $\mathrm{BH} 1$ & 37 & 0 \\
\hline pTFBH1 & FkpA-BH1 & 37 & 70 \\
\hline pTTRI & TRI & 37 & 36 \\
\hline pTTRIF & $\begin{array}{l}\text { TRI, co-expressed with } \\
\text { FkpA }\end{array}$ & 37 & 63 \\
\hline \multicolumn{4}{|c|}{ 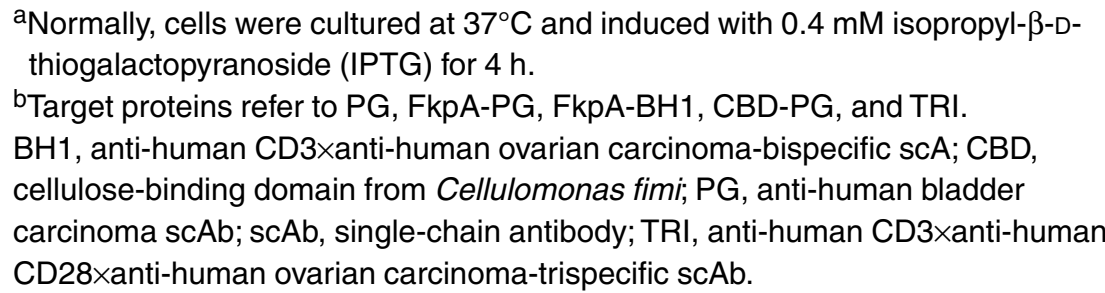 } \\
\hline
\end{tabular}

and $81 \%$ of the total amount of each target protein, respectively, expressed at $37^{\circ} \mathrm{C}$ (Table 2 ).

\section{ELISA and Western Blot Analysis Results for scAbs}

The ultimate object of FkpA-assisted expression of scAbs is to achieve biologically active products. Therefore, the antigen-binding activities of soluble FkpA-PG, FkpA-BH1, CBD-PG, and TRI, with their specific membrane antigens at different concentrations, were assayed by ELISA, and the results are shown in Figures 2C, 3B, and $4 \mathrm{~B}$ and $\mathrm{C}$, respectively. These results demonstrate that the soluble FkpA-PG, FkpA-BH1, CBD-PG, and TRI have relatively high binding affinities for their specific antigens, and the binding of the soluble target proteins with their respective antigens is specific. The immunoblotting results of the soluble TRI from co-expression with FkpA are shown in Figure 4A.

\section{In Vitro Cytotoxicity for Trispecific scAb TRI Co-Expressed with FkpA}

All the scAbs constructed previously in our laboratory were against various human carcinomas. Therefore, the in vitro cytotoxicity test is one of the most valuable evaluations. In this study, TRI-mediated in vitro cytotoxicity was measured by a colorimetric MTT-based assay, and the results are shown in Figure $4 \mathrm{D}$. Varying E/T ratios were carried out with a constant amount of $60 \mu \mathrm{g} / \mathrm{mL}$ of sonication supernatant containing TRI. Anti-human ovarian carcinoma scAb, anti-CD3 monoclonal antibody, and anti-CD28 monoclonal antibody were used as controls. In addition, 60 $\mu \mathrm{g} / \mathrm{mL}$ of sonication supernatant of $E$. coli cells harboring empty vector pTMF and PBS were used as negative controls. These in vitro cytotoxicity tests indicate that scAb TRI co-expressed with FkpA is biologically functional in vitro.

\section{DISCUSSION}

In the present work, we constructed a series of plasmids for induced expression of FkpA to assess the effects of fusion and/or co-expression on soluble and functionally active production of scAbs and their fusion proteins in $E$. coli. Our results demonstrated that FkpA is very effective for producing soluble and functional engineered antibodies in E. coli. Many attempts to improve functional expression of het- erologous proteins by overexpression with folding catalysts were carried out, but only limited success was reported (27-29). We previously tested the effects of $E$. coli Dsb proteins, a group of disulfide-forming proteins with chaperone activities, on soluble and functional expression of scAbs in E. coli and obtained excellent results (15).

As described in the Results section, a substantial portion of each fusion protein FkpA-PG, CBD-PG, FkpA-BH1, or TRI in $E$. coli was soluble and functionally active. In all ELISAs, the antigen-binding affinities of each tested scAb or its fusion protein were much higher than those of controls used. Very promisingly, the in vitro cytotoxicity assay revealed that the trispecific scAb TRI folding assisted by FkpA was able to efficiently mediate the cell lysis against specific tumor cells. Our results clearly indicate that the scAbs overexpressed with FkpA are not only soluble but also biologically active in vitro. FkpA fused to or co-expressed with scAbs provides enough molecular activity and isomerase activity for the correct folding of target proteins of interest. Therefore, it is presumed that FkpA is the main factor leading to the change of scAbs from the insoluble forms to soluble forms when expressed in E. coli. The overproduced FkpA is most likely to efficiently enhance the correct folding by preventing aggregation of folding intermediates into inclusion bodies, thus yielding correctly folded proteins. There have been reports on demonstrating the chaperone-like activity of FkpA (21). The chaperone-like activity of FkpA is probably responsible for the functional production of scAbs as a crucial factor. It is well known that some essential steps, such as the formation of correct configuration, the formation and isomerization of disulfide bonds, and the peptidyl-prolyl cis, trans isomerization, are involved in the production of functional scAbs in vivo. Actually, all the tested antibodies in our work contain several proline residues. Therefore, we proposed that the enzymatic activities of FkpA identified and still unidentified are also indispensable for the expression of biologically active scabs, since all the tested scAbs retained full antigen-binding affinities for their respective antigens and are able to kill specific tumor cells through in vitro 
cytotoxicity. In our previous work, we tested extensively the effects of the general molecular chaperones GroEL/ GroES, the excellent fusion expression partners glutathione-S-transferase (GST) and thioredoxin, and the disulfide bond-forming related proteins DsbC and $\mathrm{DsbG}$, on functional production of scAbs (15). Only DsbC and DsbG markedly enhanced the soluble and functional expression of scAbs, most probably due to their enzymatic activities as well as chaperone-like activities. In addition, high-level expression of FkpA and its fusion proteins with scAbs are not detrimental to the host cells in our experiments, suggesting that expression systems based on FkpA are applicable as well as sufficient for assisted folding expression of scAbs in E. coli.

In this report, we have described the expression with high efficiency and the marked increase of protein solubilization by overproduction of FkpA under standard culture conditions. The successful use of FkpA for the soluble and functional expression of engineered antibodies should be useful for the development of strategies for the efficient production of recombinant proteins, in general, in soluble and functional form in $E$. coli.

\section{REFERENCES}

1.Ward, E.S. 1992. Antibody engineering: the use of Escherichia coli as an expression host. FASEB J. 6:2422-2427.

2.Raag, R. and M. Whitlow. 1995. Single-chain Fvs. FASEB J. 9:73-80.

3.Cheadle, C., L.E. Hook, D. Givol, and G.A. Ricca. 1992. Cloning and expression of the variable regions of mouse myeloma protein MOPC315 in E. coli: recovery of active Fy fragments. Mol. Immunol. 29:21-30.

4.Pantoliano, M.W., R.E. Bird, S. Johnson, E.D. Asel, S.W. Dodd, J.F. Wood, and K.D. Hardman. 1991. Conformational stability, folding, and ligand-binding affinity of singlechain Fv immunoglobulin fragments expressed in Escherichia coli. Biochemistry 30:1011710125.

5.Hudson, P.J. 1998. Recombinant antibody fragments. Curr. Opin. Biotechnol. 9:395-402.

6.Knappik, A. and A. Pluckthun. 1995. Engineered turns of a recombinant antibody improve its in vivo folding. Protein Eng. 8:81-89.

7.Kipriyanov, S.M., G. Moldenhauer, and M. Little. 1997. High level production of soluble single chain antibodies in small-scale Escherichia coli cultures. J. Immunol. Methods 200:69-77.

8.Makrides, S.C. 1996. Strategies for achieving high-level expression of genes in Escherichia coli. Microbiol. Rev. 60:512-538.
9.Verma, R., E. Boleti, and A.J. George. 1998. Antibody engineering: comparison of bacterial, yeast, insect and mammalian expression systems. J. Immunol. Methods 216:165-181.

10.Humphreys, D.P., N. Weir, A. Lawson, A. Mountain, and P.A. Lund. 1996. Co-expression of human disulfide isomerase (PDI) can increase the yield of an antibody Fab' fragment expressed in Escherichia coli. FEBS Lett. 380: 194-197.

11.Knappik, A., C. Krebber, and A. Pluckthun. 1993. The effect of folding catalysts on the in vivo folding process of different antibody fragments expressed in Escherichia coli. Biotechnology (NY) 11:77-83.

12.Ward, E.S., D. Gussow, A.D. Griffiths, P.T. Jones, and G. Winter. 1989. Binding activities of a repertoire of single immunoglobulin variable domains secreted from Escherichia coli. Nature 341:544-546.

13.Skerra, A. and A. Pluckthun. 1988. Assembly of a functional immunoglobulin Fv fragment in Escherichia coli. Science 240:1038-1040.

14.Better, M., C.P. Chang, R.R. Robinson, and A.H. Horwitz. 1988. Escherichia coli secretion of an active chimeric antibody fragment. Science 240:1041-1043.

15.Zhang, Z., Z.H. Li, F. Wang, M. Fang, C.C. Yin, Z.Y. Zhou, Q. Lin, and H.L. Huang. 2002. Overexpression of DsbC and DsbG Markedly improves soluble and functional expression of single-chain fv antibodies in Escherichia coli. Protein Expr. Purif. 26:218-228.

16.Danese, P.N. and T.J. Silhavy. 1997. The sigma (E) and the Cpx signal transduction systems control the synthesis of periplasmic protein-folding enzymes in Escherichia coli. Genes Dev. 11:1183-1193.

17.Dartigalongue, C., D. Missiakas, and S. Raina. 2001. Characterization of the Escherichia coli sigma E regulon. J. Biol. Chem. 276:20866-20875

18.Missiakas, D., J.M. Betton, and S. Raina. 1996. New components of protein folding in extracytoplasmic compartments of Escherichia coli SurA, FkpA and Skp/OmpH. Mol. Microbiol. 21:871-884.

19.Ramm, K. and A. Pluckthun. 2000. The periplasmic Escherichia coli peptidylprolyl cis, trans-isomerase FkpA. II. Isomerase-independent chaperone activity in vitro. J. Biol. Chem. 275:17106-17113

20.Ramm, K. and A. Pluckthun. 2001. High enzymatic activity and chaperone function are mechanistically related features of the dimeric E. coli peptidyl-prolyl-isomerase FkpA. J. Mol. Biol. 310:485-498.

21.Arie, J.P., N. Sassoon, and J.M. Betton. 2001. Chaperone function of FkpA, a heat shock prolyl isomerase, in the periplasm of Escherichia coli. Mol. Microbiol. 39:199-210.

22.Bothmann, H. and A. Pluckthun. 2000. The periplasmic Escherichia coli peptidylprolyl cis, trans-isomerase FkpA. I. Increased functional expression of antibody fragments with and without $c i s$-prolines. J. Biol. Chem. 275: 17100-17105.

23.Sambrook, J., E.F. Fritsch, and T. Maniatis. 1989. Molecular Cloning: A Laboratory Manual, 2nd ed. CSH Laboratory Press, Cold Spring Habor, NY.

24.Heo, D.S., J.G. Park, K. Hata, R. Day, R.B.
Herberman, and T.L. Whiteside. 1990 Evaluation of tetrazolium-based semiautomatic colorimetric assay for measurement of human antitumor cytotoxicity. Cancer Res. 50: 3681-3690.

25.Zhu, Z., G. Zapata, R. Shalaby, B. Snedecor, H. Chen, and P. Carter. 1996. High level secretion of a humanized bispecific diabody from Escherichia coli. Biotechnology (NY) 14:192-196.

26.Greenwood, J.M., E. Ong, N.R. Gilkes, R.A. Warren, R.C. Miller, Jr., and D.G. Kilburn. 1992. Cellulose-binding domains: potential for purification of complex proteins. Protein Eng. 5:361-365.

27.Jeong, K.J. and S.Y. Lee. 2000. Secretory production of human leptin in Escherichia coli. Biotechnol. Bioeng. 67:398-407.

28.Kurokawa, Y., H. Yanagi, and T. Yura. 2000 Overexpression of protein disulfide isomerase DsbC stabilizes multiple-disulfide-bonded recombinant protein produced and transported to the periplasm in Escherichia coli. Appl. Environ. Microbiol. 66:3960-3965.

29.Kurokawa, Y., H. Yanagi, and T. Yura. 2001 Overproduction of bacterial protein disulfide isomerase (DsbC) and its modulator (DsbD) markedly enhances periplasmic production of human nerve growth factor in Escherichia coli. J. Biol. Chem. 276:14393-14399.

Received 30 December 2002; accepted 22 July 2003.

Address correspondence to:

Hua-liang Huang

Group 102

Institute of Genetics and Developmental Biology Academia Sinica

No. 3 Datun Rd.

Beijing 100101, China

e-mail: hlhuang@genetics.ac.cn 